\title{
Use of different combinations of enzyme complexes in broiler diets
}

\section{Utilização de diferentes combinações de complexos enzimáticos em rações para frangos de corte}

\author{
Lindolfo Dorcino dos Santos Neto'; Julyana Machado da Silva Martins²; \\ Genilson Bezerra de Carvalho3; Roberto Moraes Jardim Filho4; José Henrique \\ Stringhini5; Helder Freitas de Oliveira ${ }^{1 *}$; Marcos Barcellos Café ${ }^{5}$
}

\section{Highlights}

Use of enzymes improves weight gain and feed conversion in the pre-starter phase.

The use of enzyme complexes does not influence carcass yield.

Enzyme complexes $A$ and $B$ with $\alpha$-galactosidase improve ether extract digestibility.

\begin{abstract}
Two experiments were carried out to investigate the effect of "on top" addition of different enzyme complexes, the enzyme $\alpha$-galactosidase and three sources of the enzyme phytase available on the market, in broiler diets. In the first experiment, 1260 one-day-old Cobb $500^{\circledR}$ chicks were distributed into seven treatments in a completely randomized design (CRD) with six replicates and 30 birds/replicate. Treatments consisted of combinations of different enzyme complexes, namely, complex A (phytase, protease, xylanase, B-glucanase, cellulase, amylase, pectinase), complex B (protease and cellulase) and complex C (xylanase, amylase and protease); isolated $\alpha$-galactosidase (GAL); and three sources of phytase (P1, P2 and P3) in the diet. The treatments were formulated as follows: $T 1$ - basal diet (BD); T2 - BD + enzyme complex $A$ + enzyme complex B (BDAB); T3 - BDAB + GAL; T4 - BD + complex A + GAL; T5 - BD + complex C + P1 + GAL (BDCG); T6 - BDCG + P2; and T7 - BDCG + P3. The following variables were measured in the experimental period of 42 days: feed intake (FI), weight gain (WG), average final weight (AFW), feed conversion (FC), and carcass yield.
\end{abstract}

1 Doctoral Students of the Postgraduate Program in Animal Science, School of Veterinary and Animal Science, Universidade Federal de Goiás, UFG, Goiânia, GO, Brazil. E-mail: lindolfodorcino@hotmail.com; helder@zootecnista. com.br

2 Profa Dra of the Educational Foundation of Ituiutaba, Universidade Estadual de Minas Gerais, UEMG, Ituiutaba, MG, Brazil. E-mail: julyanamachado_zoo@hotmail.com

3 Researcher, PhD in Animal Science by the Postgraduate Program in Animal Science, School of Veterinary and Animal Science, UFG, Goiânia, GO, Brazil. E-mail: ge.nilson.bezerra@hotmail.com

${ }^{4}$ Researcher, PhD in Animal Science, Technical Director of Animal Production, São Salvador Alimentos S.A., Itaberaí, GO, Brazil. E-mail: robertomif@hotmail.com

${ }^{5}$ Profs. Drs., Postgraduate Program in Animal Science, School of Veterinary and Animal Science, UFG, Goiânia, GO, Brazil. E-mail: henrique@ufg.br; mcafe@ufg.br

* Author for correspondence

Received: July 23, 2020 - Approved: May 08, 2021 
Significant differences occurred for AFW, WG and FC in the pre-starter phase. In the second experiment, 112 Cobb $500^{\circledR}$ chicks aged 25 days were distributed into seven treatments in a CRD with four replicates and four birds/replicate. Treatments were the same as in the first experiment. Nutrient digestibility was evaluated in an experimental period of seven days. Differences were found in the metabolism coefficient of ether extract (MCEE). Dietary inclusion of enzyme complexes improves the AFW and WG of chickens from 1 to 7 days of age and MCEE in the grower phase.

Key words: Additives. Carbohydrases. Performance. Phytase. Nutrition. Protease.

\section{Resumo}

Foram realizados dois experimentos com o objetivo de avaliar o efeito da adição "on top" de diferentes complexos enzimáticos, enzima $\alpha$-galactosidade e três fontes de enzima fitase disponíveis no mercado em dietas para frangos de corte. No primeiro experimento 1260 pintos Cobb $500^{\circledR} \mathrm{com}$ um dia de idade foram distribuídos em delineamento inteiramente casualizado (DIC), sete tratamentos, seis repetições e 30 aves/repetição. Tratamentos consistiram na associação de diferentes complexos enzimáticos: complexo A (fitase, protease, xilanase, B-glucanase, celulase, amilase, pectinase); complexo B (protease e celulase); e complexo $C$ (xilanase, amilase e protease); $\alpha$-galactosidase isolada (GAL); e três fontes de fitase (F1, F2 e F3) nas rações. Em que: T1 - ração basal (RB); T2 - RB + complexo enzimático A + complexo enzimático $B$ (RBAB); T3 - RBAB + GAL; T4 - RB + complexo A + GAL; T5 - RB + complexo C + F1 + GAL (RBCG); T6 - RBCG +F2; T7 - RBCG + F3. O período experimental foi de 42 dias. Avaliaram-se: consumo de ração (CR), ganho de peso (GP), peso médio final (PMF), conversão alimentar (CA), e rendimento de carcaça. Foram observadas diferenças significativas para PMF, GP e CA na fase pré-inicial. No segundo experimento 112 pintos Cobb $500^{\circledR} \mathrm{com} 25$ dias de idade foram distribuídos em DIC, sete tratamentos, quatro repetições e quatro aves/ repetição. Os tratamentos foram os mesmos do primeiro experimento. O período experimental foi de sete dias, avaliou-se a digestibilidade dos nutrientes. Foram observadas diferenças para coeficiente de metabolizabilidade do extrato etéreo (CMEE). A inclusão de complexos enzimáticos nas rações melhora o PMF e GP de frangos de 1 a 7 dias de idade e o CMEE na fase de crescimento.

Palavras-chave: Aditivos. Carboidrases. Desempenho. Fitase. Nutrição. Protease.

\section{Introduction}

Broiler production is constantly evolving thanks to the various technological advances in the areas of breeding, nutrition, ambience, health and management. Brazil is prominent in the world poultry production scenario, leading it in chicken meat exports and ranking second in produced volume. According to Dalólio et al. (2016), among the technological mechanisms are advances in nutrition that provided the production of feed additives, which improve the assimilation of nutrients from the diet, resulting in enhanced performance.

Maize and soybean meal are the most commonly used plant ingredients in broiler diets due to their nutritional value and availability in the Brazilian market (Leite et al., 2011). However, maize, soybean meal, and other feedstuffs used in diets mostly contain antinutritional factors such as arabinoxylans, $\beta$-glucans, protease inhibitors, trypsin, phytates, saponins, lectins and others, which worsen the quality of feed and compromise nutrient digestibility by chickens (Munyaka, 
Nandha, Kiarie, Nyachoti, \& Khafipour, 2016; Carvalho et al., 2020).

Due to the high cost of feed production in poultry farming which may range from 60 to $75 \%$ of total broiler production costs (Dosković et al., 2013) , the use of exogenous enzymes is increasingly necessary, since their action on the feed can increase the digestibility and utilization of nutrients. These enzymes act directly on various substrates, in addition to destroying anti-nutritional factors present in some feedstuffs and enhancing the action of endogenous enzymes (Moura et al., 2019).

The use of enzymes in chicken diets, e.g., amylase, protease, xylanase and phytase, among others, in the form of an enzyme complex and/or in isolation, is widely accepted and disseminated by researchers, as studies have demonstrated improvements in performance, nutrient digestibility and intestinal health (Tang, Hao, Liu, Nian, \& Ru 2014; Cowieson, Lu, Ajuwon, Knap, \& Adeola, 2016; Walk, Juntunen, Paloheimo, \& Ledoux, 2019; Moura et al., 2019).

Enzymes are proteins sensitive to physico-chemical conditions that are able to bind to a substrate and remain active. In poultry farming, exogenous enzymes, which are not synthesized by the body, are commonly supplemented to complement the action of endogenous enzymes (Ribeiro, Fassani, Makiyama, \& Clemente, 2015; Keyser, Kuterna Kaczmarek, Rutkowski, \& Vanderbeke, 2016). Supplementing broiler diets with exogenous enzymes is a highly beneficial practice to reduce production costs (Alabi, Shoyombo Akpor, Oluba, \& Adeyonu, 2019).

Therefore, this study was developed to investigate the effect of "on top" addition of different enzyme complexes, the enzyme $\alpha$-galactosidase and three sources of the enzyme phytase available on the market on performance, nutrient digestibility and carcass yield of broilers from 1 to 42 days old.

\section{Material and Methods}

Two experiments were conducted at the Department of Animal Science at the School of Veterinary and Animal Science of the Federal University of Goiás (EVZ/UFG), located in Goiânia - GO, Brazil. The research project was approved by the Ethics Committee on the Use of Animals (CEUA) at UFG (approval no. 097/14).

The first experiment was conducted at the Escola Aviary at EVZ/UFG (16 ${ }^{\circ} 35^{\prime} 48.3^{\prime \prime} \mathrm{S}$, $49^{\circ} 17^{\prime} 08.8^{\prime \prime}$ W). A total of 1260 one-day-old male Cobb500 ${ }^{\circledR}$ chicks with an average weight of $36.8 \pm 0.3 \mathrm{~g}$ were distributed into seven treatments in a completely randomized design with six replicates of 30 birds each. Treatments consisted of "on top" addition of enzyme complexes, namely, complex A (phytase, protease, xylanase, B-glucanase, cellulase, amylase, pectinase), complex $B$ (protease and cellulase) and complex $C$ (xylanase, amylase and protease); the enzyme $\alpha$-galactosidase isolated (GAL); and three sources of the enzyme phytase (P1, P2 and P3), to the diets. The treatments were formulated as follows: T1 - basal diet (BD); T2 - BD + enzyme complex A + enzyme complex B (BDAB); T3 - BDAB + GAL; $\mathrm{T} 4$ - BD + complex A + GAL; T5 - BD + complex $\mathrm{C}+\mathrm{P} 1$ + GAL (BDCG); T6 - BDCG + P2; and T7 $-B D C G+P 3$.

The experimental period was 42 days, which were divided into four phases: prestarter (1 to 7 days), starter (8 to 21 days), grower (22 to 35 days) and finisher (36 to 42 
days). The experimental diets were based on maize, soybean meal and vitamin-mineral supplements and were formulated according to the nutritional requirements proposed by Rostagno et al. (2011). Tables 1, 2, 3 and 4 describe the nutritional composition and percentage of diets for all phases.

All birdswere housed in 42 experimental boxes made of plastic mesh and PVC pipes, with dimensions of $1.80 \times 1.60 \mathrm{~m}$, that were equipped with nipple drinkers, poultry feeders and rice husk litter. The boxes were located inside an industrial masonry building with clay tiles, concrete floors and screened walls. In addition, the facility was equipped with a negative ventilation system, a diesel heater, pad coolers and misters.

Water and feed were available ad libitum throughout the experimental period. Internal heating in the shed was monitored by checking the air temperature and relative humidity. Constant lighting was provided by fluorescent lamps.

Performance was evaluated on the 7th, 14th, 21st, 28th, 35th and 42nd days. The following variables were measured: feed intake (g), average weight (g), weight gain (g) and feed conversion $(\mathrm{kg} / \mathrm{kg}$ ). Feed intake $(\mathrm{g})$ was calculated per experimental unit as the weight difference between the feed provided and orts. Average weight was calculated as the total weight of the chickens divided by the number of birds in the plot. Weight gain was determined as the difference between the average initial and final weights of the birds in each studied period. Feed conversion was the result of the ratio between weight gain and feed intake. The performance variables were calculated considering the mortality rate, which was recorded daily.
To determine the yields of carcass, breast, drumsticks, wings and abdominal fat, two birds representing the average weight of the plot were selected per replicate on their $42 \mathrm{nd}$ day of age. These were fasted for eight hours and then slaughtered after stunning with an electric shock. Afterwards, the birds were weighed again and eviscerated. Subsequently, the major cuts were extracted (breast, drumsticks and wings) and the abdominal fat present in the entire cavity and adhered to the bursa was collected and weighed individually on a precision scale. Carcass yield (CY) was calculated relative to the live weight before slaughter and expressed as a percentage. The yields of the carcass parts, breast and abdominal fat were determined relative to the weight of the carcass including head and legs.

The second experiment was carried out at the Experimental Aviary at EVZ/UFG (16 ${ }^{\circ} 35^{\prime} 33.0^{\prime \prime} \mathrm{S}, 49^{\circ} 16^{\prime} 51.4^{\prime \prime} \mathrm{W}$ ) and consisted of a metabolism trial. A total of 112 male Cobb500 ${ }^{\circledR}$ chicks at 25 days of age, with an average weight of $1390.5 \pm 5 \mathrm{~g}$, were used. The birds were distributed into seven treatments in a completely randomized design with four replicates and four animals per replicate.

The same treatments tested in the first experiment were used, namely, T1 - basal diet (BD); T2 - BD + enzyme complex A + enzyme complex B (BDAB); T3 - BDAB + GAL; T4 - BD + complex A + GAL; T5 - BD + complex C + P1 + GAL (BDCG); T6 - BDCG + P2; and T7 - BDCG + P3. The experimental period was seven days, which consisted of three days of adaptation to the experimental batteries and four days that corresponded to the collection period. The metabolism trial was conducted from the 28th to the 32nd day, by the total excreta collection method (Sakomura \& Rostagno, 
Table 1

Formulae and centesimal composition of experimental diets containing enzyme complexes, $\alpha$-galactosidase and sources of phytase in the pre-starter phase (1 to 7 days)

\begin{tabular}{|c|c|c|c|c|c|c|c|}
\hline \multirow{3}{*}{ Ingredient } & \multicolumn{7}{|c|}{ Diet composition (\%) } \\
\hline & \multicolumn{7}{|c|}{ 1-7 days } \\
\hline & T1 & T2 & T3 & T4 & T5 & T6 & T7 \\
\hline Maize & 56.10 & 56.10 & 56.10 & 56.10 & 56.10 & 56.10 & 56.10 \\
\hline Soybean meal & 36.20 & 36.20 & 36.20 & 36.20 & 36.20 & 36.20 & 36.20 \\
\hline Meat meal & 4.90 & 4.90 & 4.90 & 4.90 & 4.90 & 4.90 & 4.90 \\
\hline Offal oil & 1.50 & 1.50 & 1.50 & 1.50 & 1.50 & 1.50 & 1.50 \\
\hline Complex $\mathrm{A}^{1}$ & & 0.02 & 0.02 & 0.02 & & & \\
\hline Complex B2 & & 0.05 & 0.05 & & & & \\
\hline Complex $\mathrm{C}^{3}$ & & & & & 0.01 & 0.01 & 0.01 \\
\hline$\alpha$-galactosidase ${ }^{4}$ & & & 0.05 & 0.05 & 0.05 & 0.05 & 0.05 \\
\hline Phytase $1^{5}$ & & & & & 0.01 & & \\
\hline Phytase $2^{6}$ & & & & & & 0.005 & \\
\hline Phytase $3^{7}$ & & & & & & & 0.01 \\
\hline Methionine & 0.36 & 0.36 & 0.36 & 0.36 & 0.36 & 0.36 & 0.36 \\
\hline Lysine & 0.31 & 0.31 & 0.31 & 0.31 & 0.31 & 0.31 & 0.31 \\
\hline Choline & 0.09 & 0.09 & 0.09 & 0.09 & 0.09 & 0.09 & 0.09 \\
\hline Vitamin supplement ${ }^{8}$ & 0.05 & 0.05 & 0.05 & 0.05 & 0.05 & 0.05 & 0.05 \\
\hline Mineral supplement ${ }^{9}$ & 0.10 & 0.10 & 0.10 & 0.10 & 0.10 & 0.10 & 0.10 \\
\hline Additives & 0.39 & 0.39 & 0.39 & 0.39 & 0.39 & 0.39 & 0.39 \\
\hline \multirow[t]{2}{*}{ TOTAL } & 100.00 & 100.00 & 100.00 & 100.00 & 100.00 & 100.00 & 100.00 \\
\hline & \multicolumn{7}{|c|}{ Calculated composition } \\
\hline Met. energy (Kcal/kg) & 2.980 & 2.980 & 2.980 & 2.980 & 2.980 & 2.980 & 2.980 \\
\hline Crude protein (\%) & 23.89 & 23.89 & 23.89 & 23.89 & 23.89 & 23.89 & 23.89 \\
\hline Dig. arginine (\%) & 1.49 & 1.49 & 1.49 & 1.49 & 1.49 & 1.49 & 1.49 \\
\hline Dig. met. + cys. (\%) & 0.98 & 0.98 & 0.98 & 0.98 & 0.98 & 0.98 & 0.98 \\
\hline Dig. methionine (\%) & 0.68 & 0.68 & 0.68 & 0.68 & 0.68 & 0.68 & 0.68 \\
\hline Dig. lysine (\%) & 1.33 & 1.33 & 1.33 & 1.33 & 1.33 & 1.33 & 1.33 \\
\hline Calcium (\%) & 1.00 & 1.00 & 1.00 & 1.00 & 1.00 & 1.00 & 1.00 \\
\hline Available phosphorus (\%) & 0.50 & 0.50 & 0.50 & 0.50 & 0.50 & 0.50 & 0.50 \\
\hline Sodium (\%) & 0.23 & 0.23 & 0.23 & 0.23 & 0.23 & 0.23 & 0.23 \\
\hline
\end{tabular}

T1: basal diet (BD); T2: BD + complex A + complex B (BDAB); T3: BDAB + $\alpha$-galactosidase; T4: $\mathrm{BD}+$ complex $\mathrm{A}+$ $\alpha$-galactosidase; T5: BD + complex C + phytase $1+\alpha$-galactosidase (BDCG); T6: BDCG + phytase 2; T7: BDCG + phytase 3. ${ }^{1}$ Pectinase $40,000 / \mathrm{U} \mathrm{g}^{-1}$; protease $700 / \mathrm{U} \mathrm{g}^{-1}$; phytase $300 / \mathrm{U} \mathrm{g}^{-1}$; $\beta$-glucanase $200 / \mathrm{U} \mathrm{g}^{-1}$; xylanase $100 / \mathrm{U} \mathrm{g}^{-1}$; cellulase $40 / \mathrm{U}$ $\mathrm{g}^{-1}$; amylase $30 / \mathrm{U} \mathrm{g} \mathrm{g}^{-1}$; ${ }^{2}$ rotease $7,500 / \mathrm{U} \mathrm{g}^{-1}$; cellulase $45 / \mathrm{U} \mathrm{g}^{-1} ;{ }^{3}$ endo-1,4- $\beta$-xylanase $20,000 / \mathrm{U} \mathrm{g}^{-1}$; subtilisin (protease) $40,000 / \mathrm{U} \mathrm{g}^{-1} ; \alpha$-amylase $2,000 / \mathrm{U} \mathrm{g}^{-1} ;{ }^{4} \alpha$-galactosidase $30 / \mathrm{U} \mathrm{g}^{-1} ;{ }^{5} 6$-phytase $5,000 / \mathrm{U} \mathrm{g}^{-1} ;{ }^{6} 3$-phytase $10,000 / \mathrm{FTU} \mathrm{g}^{-1}$; ${ }^{7} 6$-phytase $5,000 / \mathrm{U} \mathrm{g}^{-1}$.

${ }^{8}$ Vitamin supplement - guaranteed levels/kg of feed: selenium - $0.30 \mathrm{mg}$; vit. A - 10,000 IU; vit. D3 - 2,500 IU; vit. E - 25 mg; vit. K3 - 2 mg; vit. B1 - 2.50 mg; vit. B2 - $6.50 \mathrm{mg}$; vit. B6 - $3.50 \mathrm{mg}$; vit. B12 - $18 \mathrm{mcg}$; folic acid $-1.20 \mathrm{mg}$; pantothenic acid - 15 mg; niacin - 42 mg; biotin - 80 mcg; ethoxyquin - $166 \mathrm{mg}$; ${ }^{9}$ Mineral supplement - guaranteed levels $/ \mathrm{kg}$ of feed: manganese - 90 mg; zinc - 75 mg; iron - 60 mg; copper- 9.75 mg; iodine - 1.20 mg; growth promoter: bacitracin. 
Table 2

Formulae and centesimal composition of experimental diets containing enzyme complexes, $\alpha$-galactosidase and sources of phytase in the starter phase (8 to 21 days)

\begin{tabular}{|c|c|c|c|c|c|c|c|}
\hline \multirow{3}{*}{ Ingredient } & \multicolumn{7}{|c|}{ Diet composition (\%) } \\
\hline & \multicolumn{7}{|c|}{ 8-21 days } \\
\hline & $\mathrm{T} 1$ & $\mathrm{~T} 2$ & T3 & $\mathrm{T} 4$ & T5 & T6 & T7 \\
\hline Maize & 60.50 & 60.50 & 60.50 & 60.50 & 60.50 & 60.50 & 60.50 \\
\hline Soybean meal & 31.35 & 31.35 & 31.35 & 31.35 & 31.35 & 31.35 & 31.35 \\
\hline Meat meal & 4.10 & 4.10 & 4.10 & 4.10 & 4.10 & 4.10 & 4.10 \\
\hline Offal oil & 2.10 & 2.10 & 2.10 & 2.10 & 2.10 & 2.10 & 2.10 \\
\hline Complex $\mathrm{A}^{1}$ & & 0.02 & 0.02 & 0.02 & & & \\
\hline Complex B2 & & 0.05 & 0.05 & & & & \\
\hline Complex $\mathrm{C}^{3}$ & & & & & 0.01 & 0.01 & 0.01 \\
\hline$\alpha$-galactosidase ${ }^{4}$ & & & 0.05 & 0.05 & 0.05 & 0.05 & 0.05 \\
\hline Phytase $1^{5}$ & & & & & 0.01 & & \\
\hline Phytase $2^{6}$ & & & & & & 0.005 & \\
\hline Phytase $3^{7}$ & & & & & & & 0.01 \\
\hline Methionine & 0.33 & 0.33 & 0.33 & 0.33 & 0.33 & 0.33 & 0.33 \\
\hline Lysine & 0.30 & 0.30 & 0.30 & 0.30 & 0.30 & 0.30 & 0.30 \\
\hline Choline & 0.09 & 0.09 & 0.09 & 0.09 & 0.09 & 0.09 & 0.09 \\
\hline Vitamin supplement ${ }^{8}$ & 0.67 & 0.67 & 0.67 & 0.67 & 0.67 & 0.67 & 0.67 \\
\hline Mineral supplement ${ }^{9}$ & 0.13 & 0.13 & 0.13 & 0.13 & 0.13 & 0.13 & 0.13 \\
\hline Additives & 0.43 & 0.43 & 0.43 & 0.43 & 0.43 & 0.43 & 0.43 \\
\hline \multirow[t]{2}{*}{ TOTAL } & 100.00 & 100.00 & 100.00 & 100.00 & 100.00 & 100.00 & 100.00 \\
\hline & \multicolumn{7}{|c|}{ Calculated composition } \\
\hline Met. energy (Kcal/kg) & 3.080 & 3.080 & 3.080 & 3.080 & 3.080 & 3.080 & 3.080 \\
\hline Crude protein (\%) & 21.74 & 21.74 & 21.74 & 21.74 & 21.74 & 21.74 & 21.74 \\
\hline Dig. arginine (\%) & 1.34 & 1.34 & 1.34 & 1.34 & 1.34 & 1.34 & 1.34 \\
\hline Dig. met. + cys. (\%) & 0.90 & 0.90 & 0.90 & 0.90 & 0.90 & 0.90 & 0.90 \\
\hline Dig. methionine (\%) & 0.62 & 0.62 & 0.62 & 0.62 & 0.62 & 0.62 & 0.62 \\
\hline Dig. lysine (\%) & 1.20 & 1.20 & 1.20 & 1.20 & 1.20 & 1.20 & 1.20 \\
\hline Calcium (\%) & 0.93 & 0.93 & 0.93 & 0.93 & 0.93 & 0.93 & 0.93 \\
\hline Available phosphorus (\%) & 0.45 & 0.45 & 0.45 & 0.45 & 0.45 & 0.45 & 0.45 \\
\hline Sodium (\%) & 0.20 & 0.20 & 0.20 & 0.20 & 0.20 & 0.20 & 0.20 \\
\hline
\end{tabular}

T1: basal diet (BD); T2: $\mathrm{BD}+$ complex $\mathrm{A}+$ complex B (BDAB); T3: BDAB + $\alpha$-galactosidase; $\mathrm{T} 4: \mathrm{BD}+\mathrm{complex} \mathrm{A}+$ $\alpha$-galactosidase; T5: BD + complex C + phytase $1+\alpha$-galactosidase (BDCG); T6: BDCG + phytase 2; T7: BDCG + phytase 3 . ${ }^{1}$ Pectinase $40,000 / \mathrm{U} \mathrm{g} \mathrm{g}^{-1}$; protease $700 / \mathrm{U} \mathrm{g}^{-1}$; phytase $300 / \mathrm{U} \mathrm{g}^{-1} ; \beta$-glucanase $200 / \mathrm{U} \mathrm{g} \mathrm{g}^{-1}$; xylanase $100 / \mathrm{U} \mathrm{g}{ }^{-1}$; cellulase $40 / \mathrm{U}$ $\mathrm{g}^{-1}$; amylase $30 / \mathrm{U} \mathrm{g} \mathrm{g}^{-1}$; ${ }^{2}$ Protease $7,500 / \mathrm{U} \mathrm{g}^{-1}$; cellulase $45 / \mathrm{U} \mathrm{g}^{-1}$; ${ }^{3}$ endo-1,4- $\beta$-xylanase $20,000 / \mathrm{U} \mathrm{g}^{-1}$; subtilisin (protease) $40,000 / \mathrm{U} \mathrm{g}^{-1} ; \alpha$-amylase $2,000 / \mathrm{U} \mathrm{g}^{-1} ;{ }^{4} \alpha$-galactosidase $30 / \mathrm{U} \mathrm{g}^{-1} ;{ }^{5} 6$-phytase $5,000 / \mathrm{U} \mathrm{g}^{-1} ;{ }^{6} 3$-phytase $10,000 /$ FTU g-1; ${ }^{7} 6$-phytase $5,000 / \mathrm{U} \mathrm{g}^{-1}$.

${ }^{8}$ Vitamin supplement - guaranteed levels/kg of feed: selenium - 0.30 mg; vit. A - 10,000 IU; vit. D3 - 2,500 IU; vit. E - 25 mg; vit. K3 - 2 mg; vit. B1 - 2.50 mg; vit. B2 - 6.50 mg; vit. B6 - 3.50 mg; vit. B12 - 18 mcg; folic acid - 1.20 mg; pantothenic acid - 15 mg; niacin - 42 mg; biotin - 80 mcg; ethoxyquin - 166 mg; ${ }^{9}$ Mineral supplement - guaranteed levels/kg of feed: manganese - 90 mg; zinc - 75 mg; iron - 60 mg; copper- 9.75 mg; iodine - 1.20 mg; growth promoter: bacitracin. 


\section{Table 3}

Formulae and centesimal composition of experimental diets containing enzyme complexes, $\alpha$-galactosidase and sources of phytase in the grower phase (22 to 35 days)

\begin{tabular}{|c|c|c|c|c|c|c|c|}
\hline \multirow{3}{*}{ Ingredient } & \multicolumn{7}{|c|}{ Diet composition (\%) } \\
\hline & \multicolumn{7}{|c|}{ 22-35 days } \\
\hline & $\mathrm{T} 1$ & T2 & T3 & T4 & T5 & T6 & T7 \\
\hline Maize & 64.30 & 64.30 & 64.30 & 64.30 & 64.30 & 64.30 & 64.30 \\
\hline Soybean meal & 24.35 & 24.35 & 24.35 & 24.35 & 24.35 & 24.35 & 24.35 \\
\hline Meat meal & 3.40 & 3.40 & 3.40 & 3.40 & 3.40 & 3.40 & 3.40 \\
\hline Offal meal & 1.00 & 1.00 & 1.00 & 1.00 & 1.00 & 1.00 & 1.00 \\
\hline Feather and blood meal & 1.00 & 1.00 & 1.00 & 1.00 & 1.00 & 1.00 & 1.00 \\
\hline Offal oil & 3.20 & 3.20 & 3.20 & 3.20 & 3.20 & 3.20 & 3.20 \\
\hline Complex $\mathrm{A}^{1}$ & & 0.02 & 0.02 & 0.02 & & & \\
\hline Complex B2 & & 0.05 & 0.05 & & & & \\
\hline Complex $\mathrm{C}^{3}$ & & & & & 0.01 & 0.01 & 0.01 \\
\hline$\alpha$-galactosidase ${ }^{4}$ & & & 0.04 & 0.04 & 0.04 & 0.04 & 0.04 \\
\hline Phytase $1^{5}$ & & & & & 0.01 & & \\
\hline Phytase $2^{6}$ & & & & & & 0.005 & \\
\hline Phytase $3^{7}$ & & & & & & & 0.01 \\
\hline Methionine & 0.30 & 0.30 & 0.30 & 0.30 & 0.30 & 0.30 & 0.30 \\
\hline Lysine & 0.40 & 0.40 & 0.40 & 0.40 & 0.40 & 0.40 & 0.40 \\
\hline Choline & 0.07 & 0.07 & 0.07 & 0.07 & 0.07 & 0.07 & 0.07 \\
\hline Vitamin supplement ${ }^{8}$ & 0.93 & 0.93 & 0.93 & 0.93 & 0.93 & 0.93 & 0.93 \\
\hline Mineral supplement ${ }^{9}$ & 0.63 & 0.63 & 0.63 & 0.63 & 0.63 & 0.63 & 0.63 \\
\hline Additives & 0.42 & 0.42 & 0.42 & 0.42 & 0.42 & 0.42 & 0.42 \\
\hline \multirow[t]{2}{*}{ TOTAL } & 100.00 & 100.00 & 100.00 & 100.00 & 100.00 & 100.00 & 100.00 \\
\hline & \multicolumn{7}{|c|}{ Calculated composition } \\
\hline Met. energy (Kcal/kg) & 3.150 & 3.150 & 3.150 & 3.150 & 3.150 & 3.150 & 3.150 \\
\hline Crude protein (\%) & 18.60 & 18.60 & 18.60 & 18.60 & 18.60 & 18.60 & 18.60 \\
\hline Dig. arginine (\%) & 1.08 & 1.08 & 1.08 & 1.08 & 1.08 & 1.08 & 1.08 \\
\hline Dig. met. + cys. (\%) & 0.79 & 0.79 & 0.79 & 0.79 & 0.79 & 0.79 & 0.79 \\
\hline Dig. methionine (\%) & 0.53 & 0.53 & 0.53 & 0.53 & 0.53 & 0.53 & 0.53 \\
\hline Dig. lysine (\%) & 1.03 & 1.03 & 1.03 & 1.03 & 1.03 & 1.03 & 1.03 \\
\hline Calcium (\%) & 0.82 & 0.82 & 0.82 & 0.82 & 0.82 & 0.82 & 0.82 \\
\hline Available phosphorus (\%) & 0.40 & 0.40 & 0.40 & 0.40 & 0.40 & 0.40 & 0.40 \\
\hline Sodium (\%) & 0.19 & 0.19 & 0.19 & 0.19 & 0.19 & 0.19 & 0.19 \\
\hline
\end{tabular}

T1: basal diet (BD); T2: BD + complex A + complex B (BDAB); T3: BDAB + $\alpha$-galactosidase; T4: BD + complex A + $\alpha$-galactosidase; T5: BD + complex $C+$ phytase $1+\alpha$-galactosidase (BDCG); T6: BDCG + phytase 2; T7: BDCG + phytase 3.

${ }^{1}$ Pectinase $40,000 / \mathrm{U} \mathrm{g}^{-1}$; protease $700 / \mathrm{U} \mathrm{g}^{-1}$; phytase $300 / \mathrm{U} \mathrm{g}^{-1} ; \beta$-glucanase $200 / \mathrm{U} \mathrm{g}^{-1}$; xylanase $100 / \mathrm{U} \mathrm{g}^{-1}$; cellulase $40 / \mathrm{U} \mathrm{g}^{-1}$; amylase $30 / \mathrm{U} \mathrm{g}^{-1}$; ${ }^{2}$ Protease $7,500 / \mathrm{U} \mathrm{g}^{-1}$; cellulase $45 / \mathrm{U} \mathrm{g}^{-1}$; ${ }^{3}$ endo-1,4- $\beta$-xylanase $20,000 / \mathrm{U} \mathrm{g}^{-1}$; subtilisin (protease) $40,000 / \mathrm{U} \mathrm{g}^{-1} ; \alpha$-amylase $2,000 / \mathrm{U} \mathrm{g}^{-1} ;{ }^{4} \alpha$-galactosidase $30 / \mathrm{U} \mathrm{g}^{-1} ;{ }^{5} 6$-phytase $5,000 / \mathrm{U} \mathrm{g}^{-1} ;{ }^{6} 3$-phytase $10,000 / \mathrm{FTU} \mathrm{g}^{-1} ;{ }^{7} 6$-phytase $5,000 / \mathrm{U} \mathrm{g}^{-1}$.

${ }^{8}$ Vitamin supplement - guaranteed levels/kg of feed: selenium - $0.30 \mathrm{mg}$; vit. A - 10,000 IU; vit. D3 - 2,500 IU; vit. E - 25 mg; vit. K3 - 2 mg; vit. B1 - 2.50 mg; vit. B2 - 6.50 mg; vit. B6 - 3.50 mg; vit. B12 - 18 mcg; folic acid - 1.20 mg; pantothenic acid - $15 \mathrm{mg}$; niacin - 42 mg; biotin - 80 mcg; ethoxyquin - 166 mg; ${ }^{9}$ Mineral supplement - guaranteed levels $/ \mathrm{kg}$ of feed: manganese - $75 \mathrm{mg}$; zinc - $62.5 \mathrm{mg}$; iron - 50 mg; copper- 8.125 mg; iodine - 1.00 mg; growth promoter: bacitracin. 
Table 4

Formulae and centesimal composition of experimental diets containing enzyme complexes, $\alpha$-galactosidase and sources of phytase in the finisher phase (36 to 42 days)

\begin{tabular}{|c|c|c|c|c|c|c|c|}
\hline \multirow{3}{*}{ Ingredient } & \multicolumn{7}{|c|}{ Diet composition (\%) } \\
\hline & \multicolumn{7}{|c|}{ 36-42 days } \\
\hline & T1 & T2 & T3 & T4 & T5 & T6 & T7 \\
\hline Maize & 71.74 & 71.74 & 71.74 & 71.74 & 71.74 & 71.74 & 71.74 \\
\hline Soybean meal & 15.20 & 15.20 & 15.20 & 15.20 & 15.20 & 15.20 & 15.20 \\
\hline Meat meal & 2.53 & 2.53 & 2.53 & 2.53 & 2.53 & 2.53 & 2.53 \\
\hline Offal meal & 3.07 & 3.07 & 3.07 & 3.07 & 3.07 & 3.07 & 3.07 \\
\hline Feather and blood meal & 2.27 & 2.27 & 2.27 & 2.27 & 2.27 & 2.27 & 2.27 \\
\hline Offal oil & 2.93 & 2.93 & 2.93 & 2.93 & 2.93 & 2.93 & 2.93 \\
\hline Complex $\mathrm{A}^{1}$ & & 0.02 & 0.02 & 0.02 & & & \\
\hline Complex B2 & & 0.05 & 0.05 & & & & \\
\hline Complex $\mathrm{C}^{3}$ & & & & & 0.01 & 0.01 & 0.01 \\
\hline$\alpha$-galactosidase ${ }^{4}$ & & & 0.02 & 0.02 & 0.02 & 0.02 & 0.02 \\
\hline Phytase $1^{5}$ & & & & & 0.01 & & \\
\hline Phytase $2^{6}$ & & & & & & 0.005 & \\
\hline Phytase $3^{7}$ & & & & & & & 0.01 \\
\hline Methionine & 0.28 & 0.28 & 0.28 & 0.28 & 0.28 & 0.28 & 0.28 \\
\hline Lysine & 0.54 & 0.54 & 0.54 & 0.54 & 0.54 & 0.54 & 0.54 \\
\hline Choline & 0.10 & 0.10 & 0.10 & 0.10 & 0.10 & 0.10 & 0.10 \\
\hline Vitamin supplement ${ }^{8}$ & 0.21 & 0.21 & 0.21 & 0.21 & 0.21 & 0.21 & 0.21 \\
\hline Mineral supplement ${ }^{9}$ & 0.73 & 0.73 & 0.73 & 0.73 & 0.73 & 0.73 & 0.73 \\
\hline Additives & 0.40 & 0.40 & 0.40 & 0.40 & 0.40 & 0.40 & 0.40 \\
\hline \multirow[t]{2}{*}{ TOTAL } & 100.00 & 100.00 & 100.00 & 100.00 & 100.00 & 100.00 & 100.00 \\
\hline & \multicolumn{7}{|c|}{ Calculated composition } \\
\hline Met. energy (Kcal/kg) & 3.300 & 3.300 & 3.300 & 3.300 & 3.300 & 3.300 & 3.300 \\
\hline Crude protein (\%) & 17.40 & 17.40 & 17.40 & 17.40 & 17.40 & 17.40 & 17.40 \\
\hline Dig. arginine (\%) & 0.99 & 0.99 & 0.99 & 0.99 & 0.99 & 0.99 & 0.99 \\
\hline Dig. met. + cys. (\%) & 0.73 & 0.73 & 0.73 & 0.73 & 0.73 & 0.73 & 0.73 \\
\hline Dig. methionine (\%) & 0.48 & 0.48 & 0.48 & 0.48 & 0.48 & 0.48 & 0.48 \\
\hline Dig. lysine (\%) & 0.94 & 0.94 & 0.94 & 0.94 & 0.94 & 0.94 & 0.94 \\
\hline Calcium (\%) & 0.76 & 0.76 & 0.76 & 0.76 & 0.76 & 0.76 & 0.76 \\
\hline Available phosphorus (\%) & 0.39 & 0.39 & 0.39 & 0.39 & 0.39 & 0.39 & 0.39 \\
\hline Sodium (\%) & 0.19 & 0.19 & 0.19 & 0.19 & 0.19 & 0.19 & 0.19 \\
\hline
\end{tabular}

T1: basal diet (BD); T2: BD + complex A + complex B (BDAB); T3: BDAB + $\alpha$-galactosidase; T4: BD + complex A + $\alpha$-galactosidase; T5: BD + complex $\mathrm{C}+$ phytase $1+\alpha$-galactosidase (BDCG): T6: BDCG + phytase 2; T7: BDCG + phytase 3.

${ }^{1}$ Pectinase $40,000 / \mathrm{U} \mathrm{g}^{-1}$; protease $700 / \mathrm{U} \mathrm{g}^{-1}$; phytase $300 / \mathrm{U} \mathrm{g}^{-1} ; \beta$-glucanase $200 / \mathrm{U} \mathrm{g}^{-1}$; xylanase $100 / \mathrm{U} \mathrm{g}^{-1}$; cellulase $40 / \mathrm{U} \mathrm{g}^{-1}$; amylase $30 / \mathrm{U} \mathrm{g}^{-1} ;{ }^{2}$ Protease $7,500 / \mathrm{U} \mathrm{g}^{-1}$; cellulase $45 / \mathrm{U} \mathrm{g} \mathrm{g}^{-1}$; ${ }^{3}$ endo-1,4- $\beta$-xylanase $20,000 / \mathrm{U} \mathrm{g}^{-1}$; subtilisin (protease) $40,000 / \mathrm{U} \mathrm{g}^{-1} ; \alpha-a m y l a s e$ $2,000 / \mathrm{U} \mathrm{g}^{-1} ;{ }^{4} \alpha$-galactosidase $30 / \mathrm{U} \mathrm{g}^{-1} ;{ }^{5} 6$-phytase $5,000 / \mathrm{U} \mathrm{g}^{-1} ;{ }^{6} 3$-phytase $10,000 / \mathrm{FTU} \mathrm{g}^{-1} ;{ }^{7} 6$-phytase $5,000 / \mathrm{U} \mathrm{g}^{-1}$.

${ }^{8}$ Vitamin supplement - guaranteed levels/kg of feed: selenium - $0.30 \mathrm{mg}$; vit. A - 10,000 IU; vit. D3 - 2,500 IU; vit. E - 25 mg; vit. K3 - 2 mg; vit. B1 - 2.50 mg; vit. B2 - 6.50 mg; vit. B6 - 3.50 mg; vit. B12 - 18 mcg; folic acid - 1.20 mg; pantothenic acid - $15 \mathrm{mg}$; niacin - 42 mg; biotin - 80 mcg; ethoxyquin - 166 mg; ${ }^{9}$ Mineral supplement - guaranteed levels $/ \mathrm{kg}$ of feed: manganese - $75 \mathrm{mg}$; zinc - $62.5 \mathrm{mg}$; iron - 50 mg; copper- $8.125 \mathrm{mg}$; iodine - $1.00 \mathrm{mg}$; growth promoter: bacitracin. 
2016). The experimental diets were based on maize, soybean meal and vitamin-mineral supplements and were formulated according to the nutritional requirements proposed by Rostagno et al. (2011). Table 3 shows the nutritional composition and percentage of the diets for the grower phase.

All birds were housed in batteries with 28 experimental cages made of galvanized steel and with dimensions of $0.80 \times 0.75$ $\mathrm{m}$. The cages were equipped with drinkers, trough feeders and an excreta collection tray lined with plastic sheeting. The cages were located inside an experimental masonry shed, and ventilation was controlled through curtain management.

Water and feed were available ad libitum throughout the experimental period. Internal heating in the shed was monitored by checking the air temperature and relative humidity. Constant lighting was provided by incandescent lamps in each experimental unit.

The excreta were collected twice a day, packed in labeled plastic bags and frozen. For the chemical analysis, the samples were predried in a rectilinear forced-air oven at $55 \pm 5$ ${ }^{\circ} \mathrm{C}$. Subsequently, the samples were ground in a Wiley mill and the analyses were carried out according to the methodology proposed by Silva and Queiroz (2002). Nutrient balances were calculated as proposed by Matterson, Potter, Stutz and Singsen (1965), and the metabolizability coefficients as proposed by Batal and Parsons (2002) and Noy and Sklan (2002). The metabolizability coefficient was calculated as the percentage ratio between the retained (excretion minus intake) and the ingested amount of nutrients and energy, following Sakomura and Rostagno (2016).
Performance, carcass yield and digestibility data were evaluated by analysis of variance (ANOVA). When significant, the means were compared by Tukey's test, adopting $\alpha=$ 0.05 . The analyses were carried out using the $\mathrm{R}$ computer package.

\section{Results and Discussion}

Average final weight (AFW), average weight gain (AWG) and feed conversion (FC) differed $(P<0.05)$ between the treatment groups. The birds fed diets containing enzyme complexes A and B (T3) and $\alpha$-galactosidase in addition to them (T4) showed the highest AFW but did not differ from the other groups, except control, whose result was similar to those achieved with treatments T2, T4, T6 and T7. The same occurred for AWG, but, in addition to these treatment groups, the birds that received enzyme complex $C$ associated with $\alpha$-galactosidase and phytase 3 (T7) also showed a better index. For FC, the treatments containing enzyme complex C, $\alpha$-galactosidase (BDCG) and phytase 2 (T6), and BDCG + phytase 3 (T7) provided the best values. These results, however, did not differ those of the other treatment groups, except control, which was also similar to treatments T2, T3, T4 and T5. For feed intake, no differences were detected $(P>0.05)$. There were no differences $(P>0.05)$ between the treatments for the evaluated variables in the periods from 1 to 21 and 1 to 42 days of age (Table 5). 
Table 5

Average final weight (AFW), weight gain (AWG), feed intake (FI) and feed conversion (FC) of broilers fed diets containing enzyme complexes, the enzyme $\alpha$-galactosidase and different sources of phytase in different rearing periods

\begin{tabular}{|c|c|c|c|c|}
\hline Treatment & AFW (g) & AWG (g) & $\mathrm{FI}(\mathrm{g})$ & $\mathrm{FC}(\mathrm{kg} / \mathrm{kg})$ \\
\hline & \multicolumn{4}{|c|}{1 to 7 days } \\
\hline T1 & $164.3 b$ & $127.7 \mathrm{c}$ & 168.3 & $1.30 \mathrm{a}$ \\
\hline $\mathrm{T} 2$ & $166.4 \mathrm{ab}$ & 129.6 bc & 161.5 & $1.24 a b$ \\
\hline T3 & $174.4 \mathrm{a}$ & $135.3 \mathrm{a}$ & 166.8 & $1.22 \mathrm{ab}$ \\
\hline T4 & $171.2 \mathrm{ab}$ & $134.3 a b$ & 158.7 & $1.18 a b$ \\
\hline T5 & $174.1 \mathrm{a}$ & $136.9 a$ & 156.4 & $1.13 a b$ \\
\hline T6 & $170.4 \mathrm{ab}$ & $133.4 \mathrm{ac}$ & 147.6 & $1.10 \mathrm{~b}$ \\
\hline T7 & $171.0 \mathrm{ab}$ & $136.9 a$ & 151.9 & $1.13 b$ \\
\hline P-value & 0.044 & 0.031 & 0.739 & 0.030 \\
\hline \multirow[t]{2}{*}{ CV (\%) } & 2.67 & 2.40 & 7.33 & 7.87 \\
\hline & \multicolumn{4}{|c|}{1 to 21 days } \\
\hline $\mathrm{T} 1$ & 986.6 & 950.0 & 1290.4 & 1.33 \\
\hline T2 & 999.1 & 962.0 & 1341.5 & 1.37 \\
\hline T3 & 1010.7 & 973.5 & 1336.9 & 1.35 \\
\hline T4 & 1003.1 & 966.2 & 1328.6 & 1.33 \\
\hline $\mathrm{T} 5$ & 1022.9 & 985.7 & 1339.4 & 1.33 \\
\hline T6 & 1019.2 & 982.2 & 1314.6 & 1.32 \\
\hline T7 & 1004.9 & 968.2 & 1309.7 & 1.33 \\
\hline P-value & 0.128 & 0.133 & 0.766 & 0.796 \\
\hline \multirow[t]{2}{*}{ CV (\%) } & 3.47 & 3.59 & 2.99 & 2.93 \\
\hline & \multicolumn{4}{|c|}{1 to 42 days } \\
\hline T1 & 2895 & 2859 & 5184 & 1.74 \\
\hline T2 & 2949 & 2912 & 5257 & 1.73 \\
\hline T3 & 3002 & 2964 & 5108 & 1.65 \\
\hline $\mathrm{T} 4$ & 2899 & 2862 & 5169 & 1.70 \\
\hline T5 & 2931 & 2894 & 5222 & 1.67 \\
\hline T6 & 2931 & 2894 & 5187 & 1.68 \\
\hline $\mathrm{T} 7$ & 2922 & 2885 & 5147 & 1.65 \\
\hline P-value & 2.76 & 2.79 & 2.26 & 3.23 \\
\hline CV(\%) & 0.888 & 0.875 & 0.140 & 0.108 \\
\hline
\end{tabular}

T1: basal diet (BD); T2: BD + complex A + complex B (BDAB); T3: BDAB + $\alpha$-galactosidase; $T 4: \mathrm{BD}+$ complex $\mathrm{A}+$ $\alpha$-galactosidase; T5: BD + complex C + phytase $1+\alpha$-galactosidase (BDCG); T6: BDCG + phytase 2; T7: BDCG + phytase 3. Means followed by different letters in the columns differ by Tukey's test $(\mathrm{P}<0.05)$.

$\mathrm{CV}=$ coefficient of variation. 
Studies involving the use of enzyme complexes in broiler nutrition have shown results close to those observed in the present experiment. Similarly, Fernandes et al. (2015) evaluated the use of a complex composed of carbohydrases and protease and found that it provided a better FC than control diet. The current results corroborate the findings of Miranda, Goulart, Leite, Batista and Lima (2017), who evaluated the use of xylanase and phytase in broiler diets and reported that enzyme supplementation resulted in higher AFW and AWG in the pre-starter phase, when compared with the group fed the control diet. Likewise, Shirmohammad and Mehri (2011) observed no changes in feed intake in response to the dietary inclusion of an enzyme complex composed of carbohydrases and protease.

Physiological changes take place in the digestive tract of broilers during the first week of life. This period is followed by an increase in secretion and activity of digestive enzymes, which in turn increases nutrient degradation and availability (Dosković et al., 2013). Thus, enzyme supplementation for young animals can improve nutrient utilization, as these animals still have their digestive tract immature and their endogenous enzyme production is still low. In agreement with this, Carvalho et al. (2020) recommended the use of protease in broiler diets only in the starter phase.

Results for the period from 1 to 21 days were similar to those described by Kaczmarek et al. (2014), who studied the association of amylase and protease on the digestibility and performance of broilers in the starter phase. The authors observed that enzyme supplementation did not influence weight gain or feed conversion. Likewise, Apperson and Cherian (2017) used an enzyme complex composed of cellulase, xylanase, glucanase, $\beta$-mannanase and $\alpha$-galactosidase in broiler diets and also reported that the compound did not influence the production performance of birds in the starter phase. Yuan, Wang, Zhang and Wang (2017) associated protease and carbohydrases and described results similar to those obtained in this study for the main variables of production performance, except feed conversion, which was influenced by the inclusion of the complexes, when compared with the control group.

Our findings regarding the period from 1 to 42 days corroborate those observed by Dalólio et al. (2016). In a similar study, these authors evaluated the effect of different levels of an enzyme complex composed of phytase, protease, xylanase, $\beta$-glucanase, cellulase, amylase and pectinase in broiler diets and found that enzyme supplementation did not affect production performance. Similarly, Zou, Zheng, Zhang, Ding and Bai (2013) did not observe the influence of the use of enzymes ( $\beta$-mannanase, $\alpha$-galactosidase, xylanase and $\beta$-glucanase) in broiler diets on performance from 1 to 42 days. The researchers noticed only a downward trend in feed intake, in comparison to the animals in control group.

The enzyme-supplemented animals were expected to show superior performance, not only in the pre-starter phase, but throughout the rearing period, since, besides improving digestibility (proteins, carbohydrates, starch, fibers, among others), enzyme supplementation is known to increase endogenous production of digestive enzymes. In addition, it contributes to increasing the quality of ingredients, reducing their variability and lessening the negative effects of antinutritional factors. Nonetheless, this was not observed in this study, where the 
complexes and isolated enzymes generated superior results only in the pre-starter phase. However, these findings are in agreement with those described by other authors, as already mentioned. Leite et al. (2011) stated that there may be a relationship with enzyme/substrate specificity and, therefore, with the type of ingredient. Thus, depending on variations in the chemical composition of each feedstuff, the enzyme and/or enzyme complex used in the diets may not be able to improve this degradation, digestion and absorption.

There were no differences $(P>0.05)$ between the treatments for the yields of carcass, wings, breast, drumsticks + thighs and abdominal fat relative to the live weight of chickens at 42 days of age (Table 6).

Table 6

Yields of carcass, wings, breast, drumsticks + thighs (DTY) and abdominal fat (AF) relative to the live weight of broilers fed diets containing enzyme complexes, the enzyme $\alpha$-galactosidase and different sources of phytase at $\mathbf{4 2}$ days old

\begin{tabular}{|cccccc|}
\hline Treatment & Carcass (\%) & Wings (\%) & Breast (\%) & DTY (\%) & AF (\%) \\
\hline T1 & 75.45 & 10.35 & 40.01 & 28.54 & 1.94 \\
\hline T2 & 74.21 & 10.13 & 40.05 & 30.03 & 1.89 \\
\hline T3 & 75.24 & 9.67 & 40.71 & 30.02 & 1.77 \\
\hline T4 & 75.91 & 10.13 & 40.66 & 30.14 & 1.77 \\
\hline T5 & 74.59 & 10.04 & 40.61 & 29.82 & 1.75 \\
\hline T6 & 74.37 & 9.86 & 41.00 & 30.43 & 1.85 \\
\hline T7 & 75.40 & 9.37 & 40.77 & 29.53 & 1.76 \\
\hline P-value & 0.494 & 0.331 & 0.952 & 0.215 & 0.989 \\
\hline CV (\%) & 2.99 & 10.40 & 6.08 & 5.72 & 25.15 \\
\hline
\end{tabular}

T1: basal diet (BD); T2: $\mathrm{BD}+$ complex $\mathrm{A}+$ complex B (BDAB); T3: $\mathrm{BDAB}+\alpha$-galactosidase; $\mathrm{T4}$ : $\mathrm{BD}+$ complex $\mathrm{A}+$ $\alpha$-galactosidase; T5: BD + complex C + phytase $1+\alpha$-galactosidase (BDCG); T6: BDCG + phytase 2; T7: BDCG + phytase 3. Means followed by different letters in the columns differ by Tukey's test $(P<0.05)$.

$\mathrm{CV}=$ coefficient of variation.

Carcass yield results were similar to those published by Barbosa et al. (2018), who did not observe an effect of adding enzyme complexes similar to those used in this study in the diet of chickens on the yields of the carcass and or respective major cuts. Fernandes et al. (2015) also did not notice an effect of complexes composed of carbohydrases and protease on chicken diets regarding the yields of carcass, breast, wings and abdominal fat. Despite the similarity between the results found and those of the present study, the findings of Miranda et al. (2017) differ, as they described an increase in thigh yield and a higher drumstick weight in chickens fed enzyme complexes. Under the same approach and using treatments with enzyme complexes very similar to those tested in our study, Llamas-Moya et al. (2019) found a greater increase in the yields of carcass and breast in birds fed the diets containing enzymes, when compared with control animals. 
The improvement in the yields of carcass and major cuts was expected, since exogenous enzymes improve the efficiency of absorption of nutrients from the diet and decrease the influence of antinutritional factors. Thus, it was expected that there would be greater protein deposition for muscle formation and also a greater deposit of abdominal fat, as the diets with enzyme inclusion were not deficient. This increase in protein deposition is believed to be because these animals would be physiologically receiving a greater nutritional input. Café, Borges, Fritts and Waldroup (2002) and Law, Zulkifli, Soleimani, Liang and Awad (2018) found this increase in abdominal fat yield in broilers fed diets containing enzyme complex and the enzyme protease, respectively.
However, the results of this study, are positive, given that the deposition of abdominal fat did not increase. According to Alagawany, Attia, Ibrahim, Mahmoud and El-Sayed (2017), this increase in abdominal fat in broiler chickens is considered a waste by the poultry industry, as the abdominal fat in the carcass represents additional losses and expenses, including the treatment of processing wastewater.

There were no differences $(P>0.05)$ between the treatments for nitrogen-corrected apparent metabolizable energy (AMEn) or nutrient metabolizability coefficients, except for the metabolizability coefficient of ether extract (MCEE) $(\mathrm{P}<0.001)$ (Table 7). The diets containing complexes $\mathrm{A}$ and $\mathrm{B}$ (T2) and complex $A+\alpha$-galactosidase (T4) provided the best MCEE values.

\section{Table 7}

Metabolizability coefficients of dry matter (MCDM), nitrogen (NMC), ether extract (MCEE), nitrogencorrected apparent metabolizable energy (AMEn) and nitrogen balance (NB) of broilers fed diets containing enzyme complexes, the enzyme $\alpha$-galactosidase and different sources of phytase

\begin{tabular}{|cccccc|}
\hline Treatment & MCDM (\%) & NMC (\%) & MCEE (\%) & AMEn (kcal/kg) & NB $(\mathrm{g})$ \\
\hline T1 & 76.46 & 67.99 & $83.35 \mathrm{~b}$ & 3.120 & 43.52 \\
\hline T2 & 77.78 & 67.71 & $86.21 \mathrm{a}$ & 3.095 & 45.80 \\
\hline T3 & 75.46 & 65.12 & $82.43 \mathrm{~b}$ & 3.016 & 41.49 \\
\hline T4 & 76.46 & 68.43 & $86.21 \mathrm{a}$ & 3.035 & 49.72 \\
\hline T5 & 78.13 & 67.26 & $83.39 \mathrm{~b}$ & 3.087 & 44.37 \\
T6 & 76.35 & 67.42 & $78.79 \mathrm{c}$ & 3.045 & 44.11 \\
T7 & 74.79 & 64.58 & $78.27 \mathrm{c}$ & 2.994 & 44.12 \\
\hline P-value & 0.624 & 0.720 & $<0.001$ & 0.210 & 0.067 \\
CV (\%) & 3.49 & 5.64 & 2.45 & 2.73 & 7.36 \\
\hline
\end{tabular}

T1: basal diet (BD); T2: BD + complex A + complex B (BDAB); T3: BDAB + $\alpha$-galactosidase; T4: $B D+$ complex $A+$ $\alpha$-galactosidase; T5: BD + complex C + phytase $1+\alpha$-galactosidase (BDCG); T6: BDCG + phytase 2; T7: BDCG + phytase 3. Means followed by different letters in the columns differ by Tukey's test $(P<0.05)$.

$\mathrm{CV}=$ coefficient of variation. 
These results corroborate those found by Ipcak, Cardozo, Denli and Escobero (2019), who studied the effect of an enzyme complex composed of glucanase and xylanase and did not observe changes in the metabolizability coefficients of dry matter and crude protein or AMEn. Similarly, Kaczmarek et al. (2014) also did not observe an effect of including protease and amylase in the chicken diet on AMEn. Conversely, most studies involving the use of enzyme complexes demonstrate a superiority of these treatments when compared with control birds, fed a diet without enzyme inclusion (Abdollahi, Hosking, Ning, \& Ravindram, 2016; Amerah, Romero, Awati, \& Ravindram, 2017; Moura et al., 2019).

However, the improvement in MCEE provided by some treatments denotes improved utilization of the dietary fat, which is possibly due to a synergistic action of the enzymes used. These findings corroborate the descriptions of Leite et al. (2011), who found better fat digestibility coefficients in animals fed diets with the addition of an enzyme complex (amylase, pectinase, $\beta$-glucanase, pentosanase, cellulase, protease and phytase).

\section{Conclusion}

Enzyme complexes T3 (BDAB + $\alpha$-galactosidase), T4 (BD + complex A + $\alpha$-galactosidase), T5 (BD + complex $\mathrm{C}+$ phytase $1+\alpha$-galactosidase) and T6 (BDCG + phytase 2) can be included in broiler diets until seven days of age, when aiming to improve average final weight and weight gain. Considering the entire rearing period, the use of enzyme complexes is not recommended until the final broiler rearing stages. To improve
MCEE in the grower phase, enzyme complexes T2 (BD + complex A + complex B) and T4 (BD + complex $A+\alpha$-galactosidase) can be used as an alternative.

\section{Acknowledgments}

Thanks are extended to the Research Support Foundation of the State of Goiás (FAPEG) for the Master's fellowship grant; and to São Salvador Alimentos LTDA for technical and financial support.

\section{References}

Abdollahi, M. R., Hosking, B. J., Ning, D., \& Ravindram, V. (2016). Influence of palm kernel meal inclusion and exogenous enzyme supplementation on growth performance, energy utilization, and nutrient digestibility in young broilers. Asian-Australasian Journal of Animal Science, 29(4), 539-548. doi: 10. 5713/ ajas. 15.0224

Alabi, O. O., Shoyombo, A. J., Akpor, O. B., Oluba, O. M., \& Adeyonu, A. G. (2019). Exogenous enzymes and the digestibility of nutrients by broilers: a mini review. International Journal of Poultry Science, 18(9), 404409. doi: 10.3923/ijps.2019.404.409

Alagawany, M., Attia, A. I., Ibrahim, Z. A., Mahmoud, R. A., \& El-Sayed, S. A. (2017). The effectiveness of dietary sunflower meal and exogenous enzyme on growth, digestive enzymes, carcass traits, and blood chemistry of broilers. Environmental Science and Pollution Research International, 24(13), 12319-12327. doi: 10.1007/s11356-017-8934-4 
Amerah, A. M., Romero, L. F., Awati, A., \& Ravindran, V. (2017). Effect of exogenous xylanase, amylase, and protease as single or combined activities on nutrient digestibility and growth performance of broilers fed corn/soy diets. Poultry Science, 96(4), 807-816. doi: 10.3382/ps/pew297

Apperson, K. D., \& Cherian, G. (2017). Effect of whole flax seed and carbohydrase enzymes on gastrointestinal morphology, muscle fatty acids, and production performance in broiler chickens. Poultry Science, 96(5), 1228-1234. doi: 10.3382/ ps/pew371

Barbosa, J. A., Fo., Oliveira, J. P. F., Boas, A. D. C. V., Almeida, M., Dornellas, T., Hoffmann, A. C.,... Oba, A. (2018). Características produtivas e qualitativas de frangos de corte alimentados com diferentes complexos enzimáticos. Boletim de Indústria Animal, 75, 1-9. doi: 10.17523/ bia.2018.v75.e1427

Batal, A. B., \& Parsons, C. M. (2002). Effects of age on nutrient digestibility in chicks fed different diets. Poultry Science, 81(3), 400-407. doi: 10.1093/ps/81.3.400

Café, M. B., Borges, C. A., Fritts, C. A., \& Waldroup, P. W. (2002). Avizyme improves performance of broilers fed corn-soybean meal-based diets. Journal of Applied Poultry Research, 11(1), 29-33. doi: 10. 10 93/japr/11.1.29

Carvalho, D. P., Leandro, N. S. M., Andrade, M. A., Oliveira, H. F., Pires, M. F., Teixeira, K. A.,... Stringhini, J. H. (2020). Protease inclusion in plant- and animal-based broiler diets: performance, digestibility and biometry of digestive organs. South African Journal of Animal Science, 50(2), 291-301. doi: 10.4314/sajas.v50i2.12
Cowieson, A. J., Lu, H., Ajuwon, K. M., Knap, I., \& Adeola, O. (2016). Interactive effects of dietary protein source and exogenous protease on growth performance, immune competence and jejunal health of broiler chickens. Animal Production Science, 57(2), 252-261. doi: 10.1071/AN15523

Dalólio, F. S., Moreira, J., Vaz, D. P., Albino, L. F. T., Valadares, L. R., Pires, A. V., \& Pinheiro, S. R. F. (2016). Exogenous enzymes in diets for broilers. Revista Brasileira de Saúde e Produção Animal, 17(2), 149-161. doi: 10.1590/S1519-99402016000200003

Dosković, V., Bogosavljević-Bosković, S., Pavlovski, Z., Milošević, B., Škrbić, Z., Rakonjac, S., \& Petričević, V. (2013). Enzymes in broiler diets with special reference to protease. World's Poultry Science Journal, 69(2), 343-360. doi: 10. 1017/S0043933913000342

Fernandes, J. I. M., Bortoluzzi, C., Burin, A. M., Jr., Rorig, A., Perini, R., \& Cristo, A. B. (2015). Effect of different enzymatic supplements in diets of broilers raised at high stocking density. Journal of Veterinary Medicine and Research, 2(1), 1-4. doi: 10.47739/ veterinarymedicine.1016

Ipcak, H. H., Cardozo, P.W., Denli, M., \&Escobero, S. (2019). Effect of dietary multi-enzyme supplementation on growth performance and nutrient digestibility of broilers fed mash or pellet diets. Proceedings of the International Animal Science Conference, Cappadocia, Turkey, 11.

Kaczmarek, S. A., Rogiewicz, A., Mogielnicka, M., Rutkowski, A., Jones, R. O., \& Slominski, B. A. (2014). The effect of protease, amylase, and nonstarch polysaccharide-degrading enzyme supplementation on nutrient utilization and growth performance of 
broiler chickens fed corn-soybean mealbased diets. Poultry Science, 93(7), 17451753. doi: 10.3382/ps.2013-03739

Keyser, K., Kuterna, L., Kaczmarek, S., Rutkowski, A., \& Vanderbeke, E. (2016). High dosing NSP enzymes for total protein and digestible amino acid reformulation in a wheat/corn/soybean meal diet in broilers. Journal of Applied Poultry Research, 25(2), 239-246. doi: 10.3382/ japr/pfw006

Law, F. L., Zulkifli, I., Soleimani, A. F., Liang, J. B., \& Awad, E. A. (2018). The effects of low-protein diets and protease supplementation on broiler chickens in a hot and humid tropical environment. Asian-Australasian Journal of Animal Science, 31(8), 1291-1300. doi: 10.5713/ ajas.17.0581

Leite, P. R. S. C., Leandro, N. S. M., Stringhini, J. H., Café, M. B., Gomes, N. A., \& Jardim, R. M., Fo. (2011). Desempenho de frangos de corte e digestibilidade de rações com sorgo ou milheto e complexo enzimático. Pesquisa Agropecuária Brasileira, 46(3), 280-286. doi: 10.1590/S0100-204X2011 000300008

Llamas-Moya, S., Girdler, C. P., Shalash, S. M. M., Atta, A. M., Gharib, H. B., Morsy, E. A.,... Elmenawey, M. (2019). Effect of a multicarbohydrase containing $\alpha$-galactosidase enzyme on the performance, carcass yield, and humoral immunity of broilers fed corn-soybean meal-based diets of varying energy density. Journal of Applied Poultry Research, 29(1), 142-151. doi: 10.1016/j. japr.2019.10.001
Matterson, L. D., Potter, L. M., Stutz, M. W., \& Singsen, E. P. (1965). The metabolizable energy of feeds ingredients for chickens. Agricultural Experiment Station Research Report, (7), 1-11.

Miranda, L. M. B., Goulart, C. C., Leite, S. C. B., Batista, A. S. M., \& Lima, R. C. (2017). Farelo de algodão em dietas com ou sem suplementação de enzimas para frangos de corte. Revista Ciência Agronômica, 48(4), 690-699. doi: 10.5935/1806-6690. 20170080

Moura, F. A. S., Dourado, L. R. B., Farias, L. A., Lopes, J. B., Lima, S. B. P., \& Fernandes, M. L. (2019). Complexos enzimáticos sobre a energia metabolizável e digestibilidade dos nutrientes do milheto para frangos de corte. Arquivo Brasileiro de Medicina Veterinária e Zootecnia, 71(3), 990-996. doi: 10.1590/1678-4162-10021

Munyaka, P. M., Nandha, N. K., Kiarie, E., Nyachoti, C. M., \& Khafipour, E. (2016). Impact of combined $\beta$-glucanase and xylanase enzymes on growth performance, nutrients utilization and gut microbiota in broiler chickens fed corn or wheat-based diets. Poultry Science, 95(3), 528-540. doi: 10.3382/ps/pev333

Noy, Y., \& Sklan, D. (2002). Nutrient use in chicks during the first week posthatch. Poultry Science, 81(3), 391-399. doi: 10.1093/ps/ 81.3.391

Ribeiro, J. S., Fassani, E. J., Makiyama, L., \& Clemente, A. H. S. (2015). Suplementação de enzimas amilase, fitase e protease para codornas japonesas em postura. Boletim de Indústria Animal, 72(2), 163-169. doi: 10.17523/bia.v72n2p163 
Rostagno, H. S., Albino, L. F. T., Donzele, J. L., Gomes, P. C., Oliveira, R. F., Lopes, D. C.,... Euclides, R. F. (2011). Tabelas brasileiras para aves e suínos: composição de alimentos e exigências nutricionais. Viçosa: Editora da Universidade Federal de Viçosa.

Sakomura, N. K., \& Rostagno, H. S. (2016). Métodos de pesquisa em nutrição de monogástricos. São Paulo: Editora FUNEP.

Shirmohammad, F., \& Mehri, M. (2011). Effects of dietary supplementation of multienzyme complex on the energy utilization in rooster and performance of broiler chicks. African Journal of Biotechnology, 10(38), 7541-7547. doi: 10.5897/AJB10. 2260

Silva, D. J., \& Queiroz, A. C. (2002). Análise de alimentos: métodos químicos e biológicos. Viçosa, MG: Editora da Universidade Federal de Viçosa.

Tang, D., Hao, S., Liu, G., Nian, F., \& Ru, Y. (2014). Effects of maize source and complex enzymes on performance and nutrient utilization of broilers. Asian-Australasian Journal of Animal Science, 27(12), 17551762. doi: 10.5713/ajas.2014.14255
Walk, C. L., Juntunen, K., Paloheimo, M., \& Ledoux, D. R. (2019). Evaluation of novel protease enzymes on growth performance and nutriente digestibility of poultry: enzyme dose response. Poultry Science, 98(11), 5525-5532. doi: 10.3382/ ps/pez299

Yuan, L., Wang, M., Zhang, X., \& Wang, Z. (2017). Effects of protease and non-starch polysaccharide enzyme on performance, digestive function, activity and gene expression of endogenous enzyme of broilers. Plos One, 12(3), 1-13. doi: 10.13 71/journal.pone.0173941

Zou, J., Zheng, P., Zhang, K., Ding, X., \& Bai, S. (2013). Effects of exogenous enzymes and dietary energy on performance and digestive physiology of broilers. Journal of Animal Science and Biotechnology, 4(14), 1-9. doi: 10.1186/2049-1891-4-14 
